\section{Synthesis of ultrasound contrast agents: characteristics and size distribution analysis (secondary publication)}

\author{
Hak Jong Lee ${ }^{1,2}$, Tae-Jong Yoon ${ }^{3}$, Young II Yoon ${ }^{1}$ \\ 'Program in Nano Science and Technology, Department of Transdisciplinary Studies, Seoul \\ National University Graduate School of Convergence Science and Technology, Seoul; \\ ${ }^{2}$ Department of Radiology, Seoul National University Bundang Hospital, Seoul National \\ University College of Medicine, Seongnam; ${ }^{3}$ Department of Applied Bioscience, CHA \\ University, Pocheon, Korea
}

Purpose: The purpose of this study was to establish a method for ultrasound (US) contrast agent synthesis and to evaluate the characteristics of the synthesized US contrast agent.

Methods: A US contrast agent, composed of liposome and sulfur hexafluoride $\left(\mathrm{SF}_{6}\right)$, was synthesized by dissolving $21 \mu \mathrm{mol}$ 1,2-dihexadecanoyl-sn-glycero-3-phosphocholine (DPPC, $\mathrm{C}_{40} \mathrm{H}_{80} \mathrm{NO}_{8} \mathrm{P}$ ), $9 \mu \mathrm{mol}$ cholesterol, and $1.9 \mu \mathrm{mol}$ of dihexadecylphosphate (DCP, $\left.\left[\mathrm{CH}_{3}\left(\mathrm{CH}_{2}\right) 150\right]_{2} \mathrm{P}(\mathrm{O}) \mathrm{OH}\right)$ in chloroform. After evaporation in a warm water bath and drying for 12-24 hours, the contrast agent was synthesized using the sonication process by the addition of a buffer and $\mathrm{SF}_{6}$ gas. The size distribution of the bubbles was analyzed using dynamic light scattering measurement methods. The degradation curve was evaluated by assessing the change in the number of contrast agent bubbles using light microscopy immediately, 12, 24, 36, 48, 60,72 , and 84 hours after synthesis. The echogenicity of the synthesized microbubbles was compared with commercially available microbubbles (SonoVue, Bracco).

Results: contrast agent was synthesized successfully using an evaporation-drying-sonication method. Most bubbles had a mean diameter of $154.2 \mathrm{~nm}$ and showed marked degradation 24 hours after synthesis. Although no statistically significant differences were observed between SonoVue and the synthesized contrast agent, a difference in echogenicity was observed between the synthesized contrast agent and saline $(\mathrm{P}<0.01)$.

Conclusion: We successfully synthesized a US contrast agent using an evaporation-dryingsonication method. These results may help future research in the fields of anticancer drug delivery, gene delivery, targeted molecular imaging, and targeted therapy.

Keywords: Ultrasound; Contrast media; Synthesis; Drug delivery systems; Radiotherapy, image guided

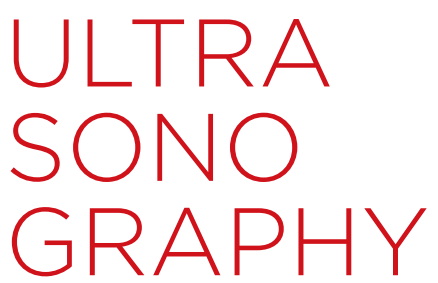

\section{ORIGINAL ARTICLE}

https://doi.org/10.14366/usg.17014 pISSN: 2288-5919 • elSSN: 2288-5943 Ultrasonography 2017;36:378-384

Received: January 26, 2017 Revised: February 10, 2017 Accepted: February 14, 2017

Correspondence to: Hak Jong Lee, MD, PhD, Department of Radiology, Seoul National University Bundang Hospital, Seoul National University College of Medicine and Program in Nano Science and Technology, Department of Transdisciplinary Studies, Seoul National University Graduate School of Convergence Science and Technology, 82 Gumi-ro 173beon-gil, Bundang-gu, Seongnam 13620, Korea

Tel. +82-31-787-7605

Fax. +82-31-787-4011

E-mail: hakjlee@snu.ac.kr

This article is an English version of "Synthesis of ultrasound contrast agents: characteristics and size distribution analysis [Korean]" published in the Journal of Korean Society of Ultrasound in Medicine in March 2013.

This is an Open Access article distributed under the terms of the Creative Commons Attribution NonCommercial License (http://creativecommons.org/ licenses/by-nc/3.0/) which permits unrestricted noncommercial use, distribution, and reproduction in any medium, provided the original work is properly cited.

Copyright (C) 2017 Korean Society of Ultrasound in Medicine (KSUM)

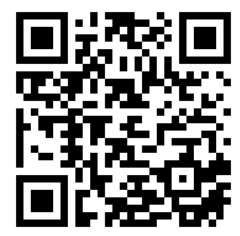

Please cite the original article as: Lee HJ, Yoon TJ, Yoon YI. Synthesis of ultrasound contrast agents: characteristics and size distribution analysis. J Korean Soc Ultrasound Med. 2013;32(1):59-65. 


\section{Introduction}

Ultrasound (US) agents used clinically, also referred to as microbubbles, are about 1-7 $\mu \mathrm{m}$ in size, and consist of an outer shell and an inner core filled with gas $[1,2]$. The outer shell is composed of either phospholipids or denatured albumin while the inner core is composed of denatured albumin that is $20-200 \mathrm{~nm}$ in thickness or phospholipids and is filled with sulfur hexafluoride $\left(\mathrm{SF}_{6}\right)$ or perfluoropropane gas [3].

These microbubbles can act as intravascular contrast agents owing to their inner gas core and can be used to deliver drugs or gene therapy products. When the frequency of a US transducer is at $1 \mathrm{MHz}$ and its mechanical index (MI) between 0.1 and 0.3 , microbubbles exhibit nonlinear oscillation and give rise to harmonic backscattering, which allows them to act as US contrast agents within blood vessels by producing signals. At an $\mathrm{Ml}$ of 0.3 or greater, microbubbles are destroyed; in those cases, they are used for disruption-replenishment, in which existing US agents are removed and new microbubbles are introduced. Temporary changes in the transparency of the cell membrane during disruptionreplenishment have been previously reported [1]. This phenomenon, called sonoporation, allows microbubbles to deliver drugs and gene therapy products. Research on drug and gene therapy product delivery using microbubbles has been actively conducted in recent years [4-6]. A good understanding of the synthesis and properties of US contrast agents is necessary when studying delivery of drugs and gene therapy products by these contrast agents. In this study, we propose a method for synthesizing US contrast agents and perform analyses on microbubble size and other properties.

\section{Materials and Methods}

The present study was performed over a period of three and a half years (from January 2009 to April 2012). The first year was spent on establishing the methodology for the synthesis of US contrast agents. In the subsequent years, we analyzed properties of the synthesized US contrast agent. The synthesis of the US contrast agent involved the following four steps: preparation of a phospholipid solution, evaporation and freeze-drying, microbubble synthesis, and microbubble size control (Fig. 1). We modified and used the method of synthesis used by Mortazavi et al. [7]. Briefly, $21 \mu \mathrm{mol}$ of 1,2-dihexadecanoyl-sn-glycero-3-phosphocholine (DPPC, $\mathrm{C}_{40} \mathrm{H}_{80} \mathrm{NO}_{8} \mathrm{P}$, Avanti, Alabaster, AL, USA), $9 \mu \mathrm{mol}$ of cholesterol (Sigma-Aldrich Korea, Yongin, Korea), and $1.9 \mu \mathrm{mol}$ of dihexadecylphosphate $\left(\mathrm{DCP},\left[\mathrm{CH}_{3}\left(\mathrm{CH}_{2}\right) 150\right]_{2} \mathrm{P}(\mathrm{O}) \mathrm{OH}\right)$, SigmaAldrich Korea) were dissolved in chloroform, which contributed to the formation of microbubble shells, an increase in the stability of the shells, and the prevention of clustering among microbubbles by distributing negative charges, respectively. Chloroform was removed by drying the solution in a rotary evaporator (SB-1100, EYELA, Tokyo, Japan) at $35^{\circ} \mathrm{C}$ for 5 minutes followed by freezedrying at $-45^{\circ} \mathrm{C}$ for 24 hours. A film with an even distribution of the aforementioned three components was then synthesized. Lastly, $6 \mathrm{~mL}$ of a buffer solution (phosphate-buffered saline, Life Technologies, California, CA, USA) and sulfur hexafluoride gas ( $\mathrm{SF}_{6}$, Dong-A Industry Gas, Seoul, Korea) were added to a flask containing the film and sonication was performed by using an ultrasonic bath (JAC 4020P, KODO, Seoul, Korea) to synthesize microbubbles. To ensure the microbubbles were uniform in size, a solution containing the microbubbles was extruded through a porous polycarbonate film with a pore size of $200 \mathrm{~nm}$ (Avanti Mini-Extruder, Avanti).

\section{Analysis of Microbubble Enhancement Time and Size}

The size of the synthesized US contrast agent was measured using the dynamic light scattering measurement method and the mean and standard deviation of the measurements were calculated.
DPPC, cholesterol, DCP in chloroform

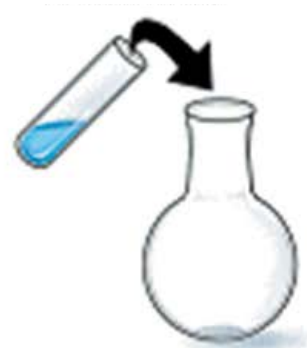

PBS buffer $\mathrm{SF}_{6}$ gas

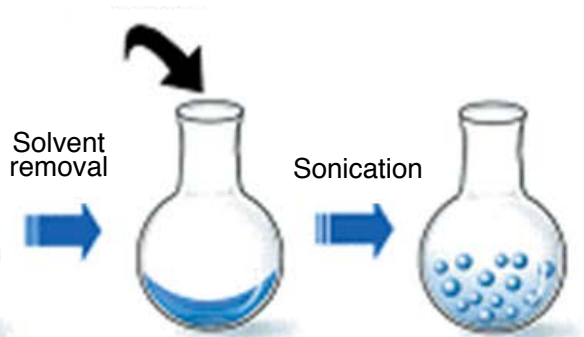

Polycarbonate membrane (for extrusion)
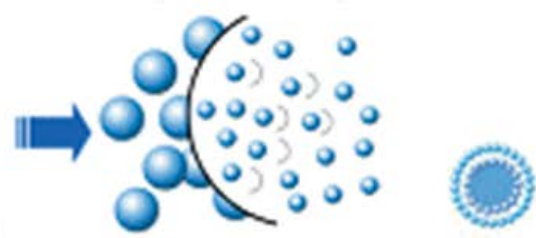

Fig. 1. Schematic drawing showing the procedure about synthesis of ultrasound contrast agents. The procedure consists of four steps: preparation of phospholipid solution, evaporation/freeze-drying, formation of bubbles, and regulation of bubble size. DPPC, 1,2-dihexadecanoyl-sn-glycero-3-phosphocholine; DCP, dihexadecylphosphate; PBS, phosphated buffered saline. 
The number of microbubbles in a sample containing $20 \mu \mathrm{L}$ of the synthesized US contrast agent was counted using a method that follows the same principle as cell counting. The synthesized US contrast agent was placed in a $20 \mathrm{~mL}$ container and left at room temperature. Each sample containing $20 \mu \mathrm{L}$ of the synthesized US contrast agent was placed in a cell counter immediately, 12, 24, 36, $48,60,72$, and 84 hours after the synthesis to study a pattern of changes in the number of microbubbles. To ensure even distribution of microbubbles within the solution, the solution was shaken 2-3 times before each sampling.

\section{US Imaging and Analysis Using US Contrast Agents}

US imaging using US contrast agents was performed by a radiologist with 13 years of experience in ultrasonography (H.J.L.). A tube measuring $3 \mathrm{~mm}$ in diameter was immersed in a container containing $200 \mathrm{~mL}$ of water and a target substance was run through the tube for image analysis.

All US imaging was performed with a 5-12 MHz broadband linear transducer (Philips, Bothell, WA, USA), and iU22 US scanner (Philips). Contrast-enhanced US images were obtained by performing pulse inversion harmonic imaging at an $\mathrm{Ml}$ of 0.07 .

For the control group, images were obtained by slowly introducing $2 \mathrm{~mL}$ of saline solution into the tube. The intensities of the solution were compared to that of a commercially available contrast agent (SonoVue, Bracco, Milan, Italy). All contrast-enhanced images were saved on a computer and analyzed with Image J software (http:// rsbweb.nih.gov/ij/), which is a public image processing program. To analyze the intensities of the contrast agents on the images, each image was enlarged by $150 \%$ and 20 circular regions of interest (ROI), consisting of 70 pixels each, were marked on the inside of the tube in which the contrast agents were present. The means and standard deviations of the intensities measured outside the ROI were calculated.

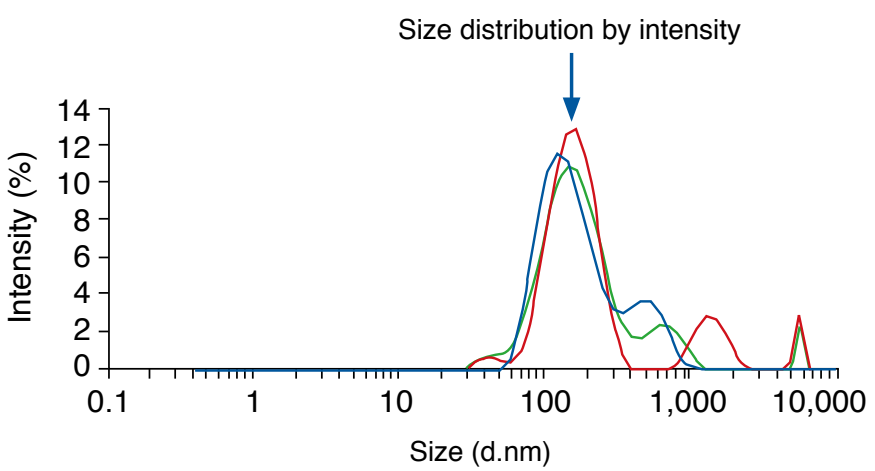

Fig. 2. Size distributions of ultrasound contrast agents. The results from dynamic light scattering measurement show that the size of first peak is about $154.2 \mathrm{~nm}$ (arrow).
The intensities of the synthesized US contrast agent, SonoVue (Bracco), and the saline solution were analyzed by performing analysis of variance (ANOVA) and a posthoc analysis. To reduce type 1 errors in the posthoc analysis, the level of statistical significance was set at less than or equal to 0.01 . SPSS version 10 (SPSS Inc., Chicago, IL, USA) was used for the statistical analysis.

\section{Results}

A US contrast agent was successfully synthesized through the aforementioned freeze-drying-sonication process (Fig. 1). Fig. 2 shows size distributions of US contrast agents on a graph. The largest distribution can be seen at the first peak, at $154.2 \mathrm{~nm}$. Fig. 3 illustrates the decrease in the number of bubbles in the synthesized US contrast agents over time. The number drastically decreased from 24 hours after synthesis onward (Fig. 3). Fig. 4 shows contrastenhanced images that were obtained by injecting US contrast agents into a model that we produced. Fig. 5 shows how the intensities of the contrast agents were actually measured by using Image I software. When the intensities of the contrast agents were compared, no statistically significant difference was found between SonoVue, a clinically used US contrast agent, and the synthesized contrast agent in terms of their echogenicity (Fig. 6). Table 1 shows echo measurements of SonoVue, the synthesized contrast agent,

Table 1. Mean and standard deviation of echogenicity on ultrasound contrast study images

\begin{tabular}{lccc}
\hline & SonoVue & $\begin{array}{c}\text { Synthesized } \\
\text { contrast agent }\end{array}$ & $\begin{array}{c}\text { Normal } \\
\text { saline }\end{array}$ \\
\hline Mean & 153.3 & 147.6 & 14.1 \\
Standard deviation & 17.1 & 13.5 & 1.9 \\
\hline
\end{tabular}

Degradation of ultrasound contrast agent

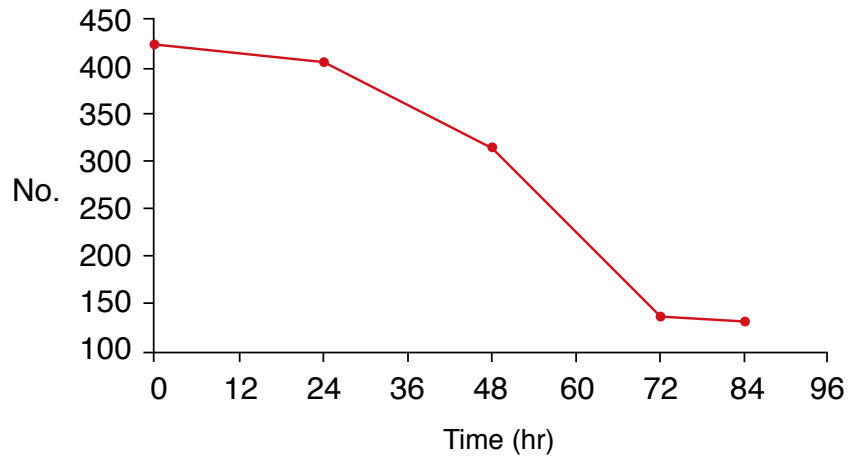

Fig. 3. Degradation of synthesized ultrasound contrast agent. The number of bubbles seen on light microscopy shows marked degradation since 24 hours after synthesis, which means that the optimal time limit of use is within 24 hours. 


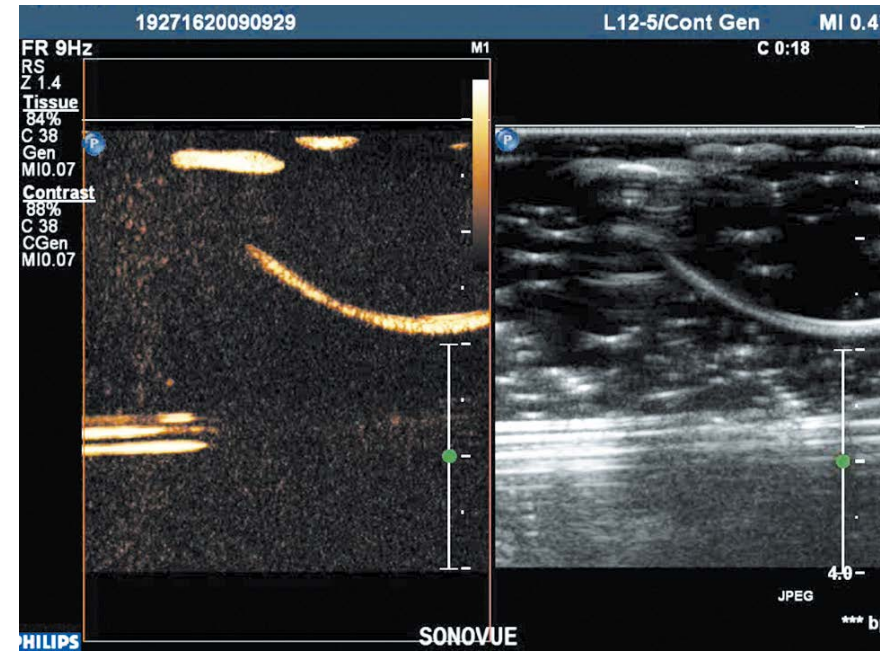

A

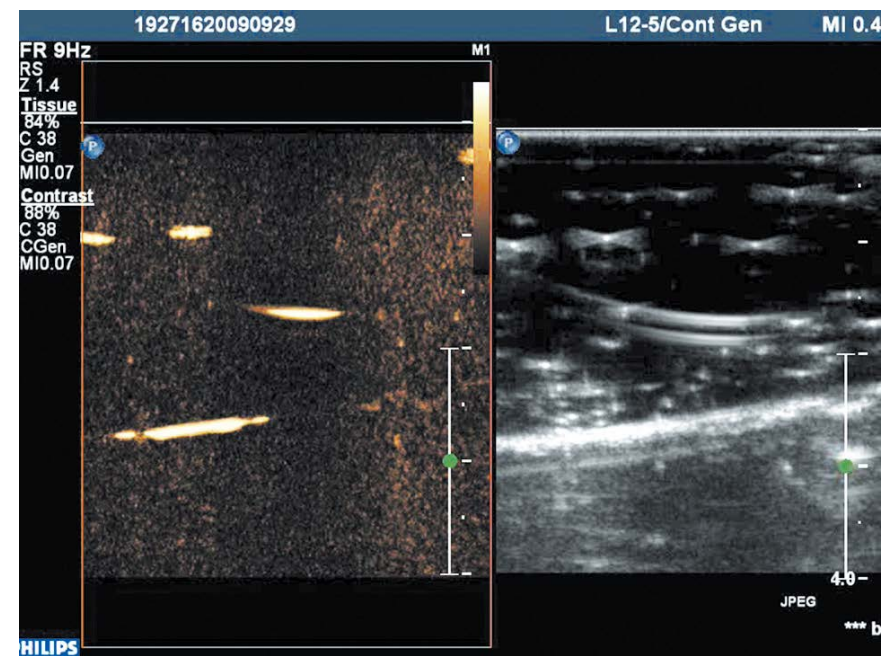

C

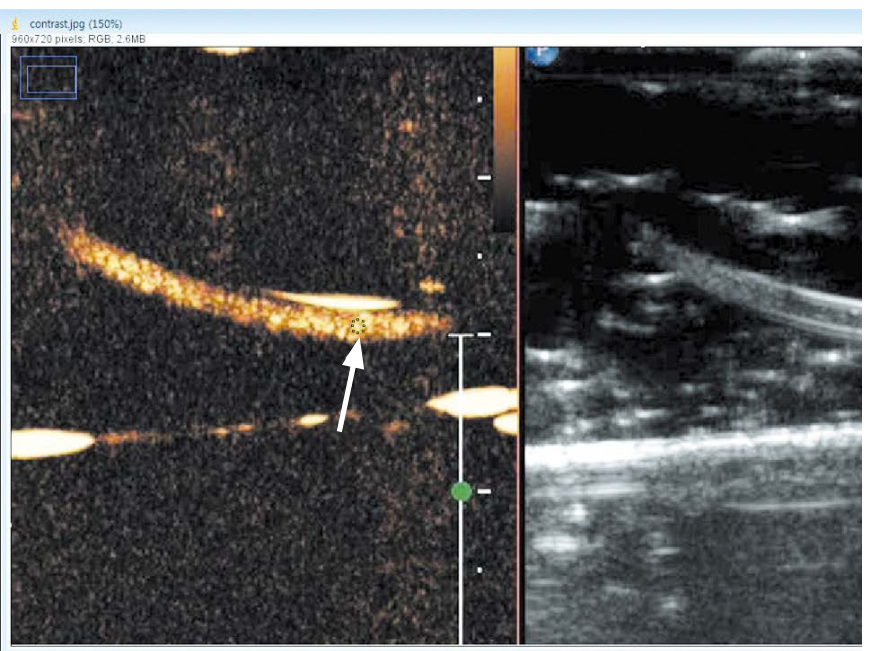

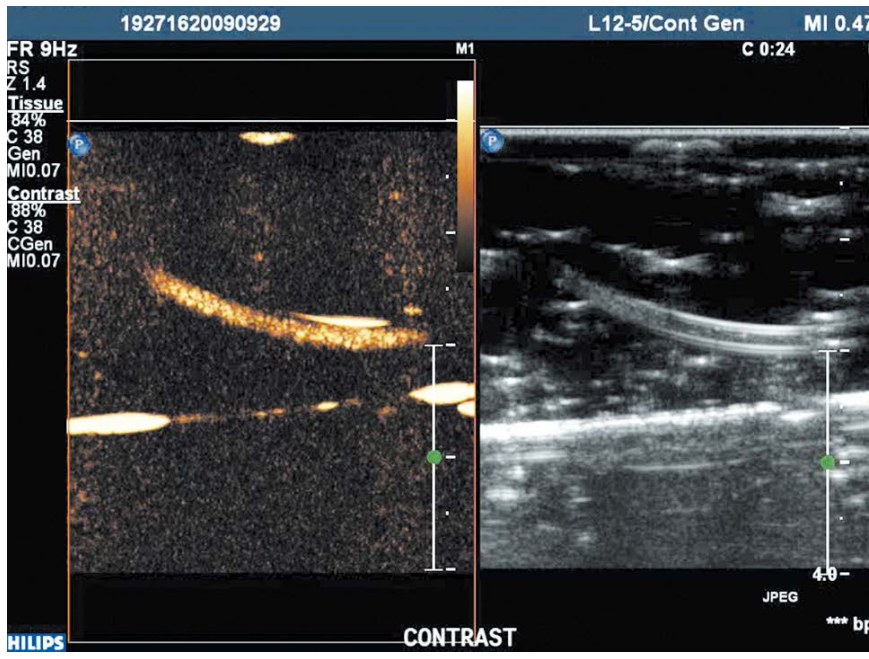

B

Fig. 4. The comparison of ultrasound contrast agent images of SonoVue (A), synthesized contrast agent (B), and normal saline (C).

The echogenicity of our synthesized contrast agent is similar to that of SonoVue. Also note that the echogenicity of saline is very low.
Fig. 5. The method showing how to measure the intensity from the ultrasound images using Image J. Twenty regions of interest (ROIs) were drawn in each images, the mean and standard deviation of intensities were calculated. Arrow in figure shows one example of ROl located in the tube. 


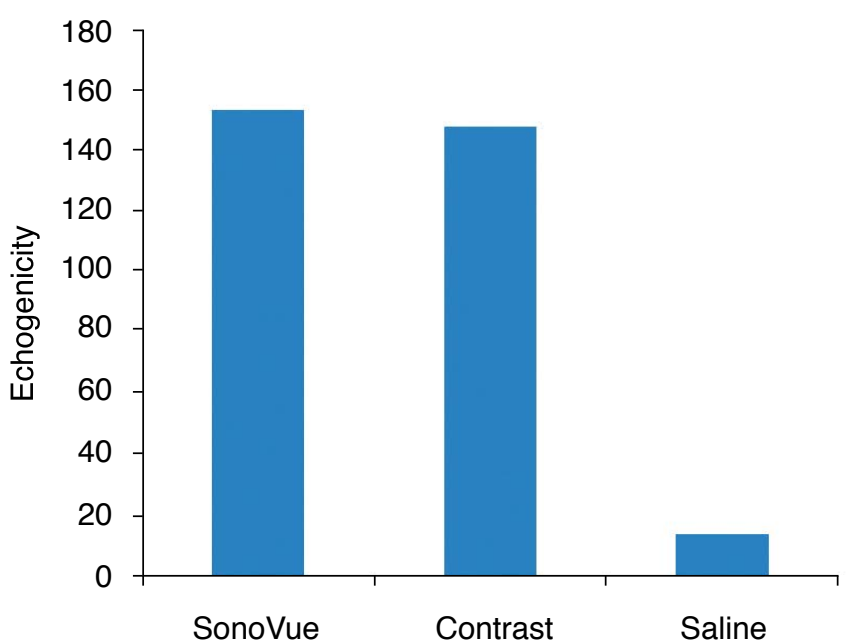

Fig. 6. Graph showing the echogenicity of SonoVue, synthesized contrast agent, and saline. Although there was no significant difference between SonoVue and synthesized contrast agent, there was difference in echogenicity between synthesized contrast agent and saline $(P<0.01)$.

and the saline solution. In the ANOVA of echogenicity, statistically significant differences were found between SonoVue, the synthesized US contrast agent, and the saline solution $(P<0.001)$. In the posthoc analysis of echogenicity, no statistically significant difference was found between SonoVue and the synthesized US contrast agent, while a statistically significant difference was observed between SonoVue and the saline solution and between the synthesized US contrast agent and the saline solution $(P<0.01)$.

\section{Discussion}

The recent development of US contrast agents has drawn attention to their use in quantification of angiogenesis and in US imaging to assess tumor angiogenesis at a molecular level and in a noninvasive way [8].

US molecular imaging has superior temporal and spatial resolutions compared to other molecular imaging techniques, is noninvasive, shows images in real time, is relatively cheap to perform, and does not use radiation. Because of these advantages, it is expected that US molecular imaging will contribute significantly to preclinical research on tumor imaging and development of novel drugs and that its clinical application will expand in the future.

US contrast agents show potential for development in many aspects because of their properties. A typical example of the properties that make US contrast agents have such potential is that US contrast agents enable noninvasive assessment of vascularity. Pysz et al. [9] have recently assessed and monitored tumor vascularity by performing contrast-enhanced US imaging, and Deshpande et al. [10] have assessed tumor angiogenesis and associated markers by using targeted microbubbles and US imaging. Willmann et al. [11] injected microbubbles bound to anti-vascular endothelial growth factor receptor 2 antibodies and those without the antibodies to murine tumor models to analyze the ability of the contrast microbubbles to track tumor blood cells. They reported that the US contrast agent bound to the antibodies showed significantly higher adherence to tumor blood cells than the control microbubbles [11]. Ellegala et al. [12] detected tumor angiogenesis by using microbubbles bound to antibodies that associate with $a_{v}$-integrin, which is expressed in the endothelial cells of new blood vessels in a tumor, and reported that information regarding the volume and velocity of blood obtained through this technique will provide further biological information about tumor angiogenesis.

US contrast agents have great application potential because of their ability to locally deliver drugs or gene therapy products within cells, which varies on the permeability of the cell membrane that forms when microbubbles rupture. Deckers et al. [13] reported on a fluorescent reporter that can be used to assess local delivery of drugs by US contrast agents. Tinkov et al. [4] reported a 12fold higher tissue concentration of a drug when a contrast agent was used in a living model as opposed to when it was not used. Research on methodologies on effective delivery of gene therapy drugs using contrast microbubbles has drawn much attention. Use of synthetic small interfering RNA (siRNA) is one of the methods of inhibiting or controlling gene expression. Carson et al. [5] recently published their research on delivery of siRNA within cancer cells using US and microbubbles in murine squamous cell tumor models.

All of these studies on US-mediated drug delivery may hold great potential for research on drug delivery through the blood-brain barrier (BBB). McDannold et al. [14] proved that temporary disruption of the BBB can be induced by use of US and microbubbles. Yang et al. [6] increased drug delivery by interleukin-4 receptor-targeted liposomal doxorubicin by using US in brain tumor animal models. Research on drug delivery through the BBB using US and microbubbles is expected to draw much attention in the future.

The aim of the present study was to directly synthesize microbubbles and analyze their properties. In order to study the delivery of various drugs and gene therapy products by US contrast agents, microbubbles containing these drugs first need to be synthesized. The method of synthesis may vary depending on the type and properties of the substance to be included in microbubbles. Therefore, the first step in research on drug and gene delivery should involve acquiring an ability to directly synthesize microbubbles. Based on this ability, the methods of synthesis of US contrast agents containing drugs or gene therapy products for target diseases can 
be subsequently developed.

In this study, the size of the synthesized microbubble was approximately $150 \mathrm{~nm}$ at the first peak. This is considered to be very small when compared to the size of microbubbles commonly used clinically, which are usually in the micrometer range. Therefore, the echogenicity of the synthesized US contrast agent was lower than that of commercially available contrast agents, such as SonoVue. However, when considering the fact that the gaps between the endothelial cells, through which substances can enter the interstitium from blood vessels, are few hundred nanometers in size, the synthesized US contrast agent may be more effective at delivering drugs or gene therapy products. While the performance of US contrast agents and the quantity of drugs or gene therapy products that they can deliver increase as the bubble size increases to the micrometer range, this may limit their selectiveness for target cells. On the other hand, while US contrast agents with bubbles in the nanometer range may have poorer performances and carry smaller amounts of drugs or gene therapy products in each bubble, they are more likely to reach target cells and approximate the concept of targeted drug delivery. Suitable strategies are required to select the appropriate approach according to the target diseases or tissues to be transplanted. Studies on this topic are, therefore, needed for each specific disease or organ.

In conclusion, US contrast agents are fascinating as that they can be used as a means to deliver drugs and gene therapy products under US image guidance. In order to study US contrast agents as drug carriers, various substances should be incorporated into the microbubbles. To do this, direct methods of US contrast agent synthesis and modification must be developed. In this study, we were able to synthesize a US contrast agent that exhibited a similar degree of echogenicity as an existing US contrast agent. We believe that this synthetic US contrast agent will become a basic prototype for drugs and gene therapy product carriers. Furthermore, our findings will promote research in various fields including anticancer drug delivery, siRNA or microRNA delivery, targeted molecular imaging, and targeted therapy.

ORCID: Hak Jong Lee: http://orcid.org/0000-0003-0858-7873; Tae-Jong Yoon: http:// orcid.org/0000-0002-0809-8319; Young II Yoon: http://orcid.org/0000-0001-99574896

\section{Conflict of Interest}

Hak Jong Lee is the co-founder of IMGT Co. Ltd. All remaining authors have declared no conflicts of interest.

\section{Acknowledgments}

The present study was funded by the Ministry of Education, Science, and Technology through basic research support provided by the National Research Foundation of Korea in 2012. This study was also supported by a Philips Scholarship Fund (2011) through the Korean Society of Ultrasound in Medicine.

\section{References}

1. Burns PN, Wilson SR. Microbubble contrast for radiological imaging: 1. Principles. Ultrasound Q 2006;22:5-13.

2. Quaia E. Microbubble ultrasound contrast agents: an update. Eur Radiol 2007;17:1995-2008.

3. Quaia E. Physical basis and principles of action of microbubblebased contrast agents. In: Quaia E, ed. Contrast media in ultrasonography. Berlin: Springer, 2005;15-30.

4. Tinkov S, Coester C, Serba S, Geis NA, Katus HA, Winter G, et al. New doxorubicin-loaded phospholipid microbubbles for targeted tumor therapy: in-vivo characterization. J Control Release 2010;148:368-372.

5. Carson AR, McTiernan CF, Lavery L, Grata M, Leng X, Wang J, et al. Ultrasound-targeted microbubble destruction to deliver siRNA cancer therapy. Cancer Res 2012;72:6191-6199.

6. Yang FY, Wong TT, Teng MC, Liu RS, Lu M, Liang HF, et al. Focused ultrasound and interleukin-4 receptor-targeted liposomal doxorubicin for enhanced targeted drug delivery and antitumor effect in glioblastoma multiforme. J Control Release 2012;160:652658.

7. Mortazavi SM, Mohammadabadi MR, Khosravi-Darani K, Mozafari MR. Preparation of liposomal gene therapy vectors by a scalable method without using volatile solvents or detergents. J Biotechnol 2007:129:604-613.

8. Deshpande N, Pysz MA, Willmann JK. Molecular ultrasound assessment of tumor angiogenesis. Angiogenesis 2010;13:175188.

9. Pysz MA, Foygel K, Panje CM, Needles A, Tian L, Willmann JK. Assessment and monitoring tumor vascularity with contrastenhanced ultrasound maximum intensity persistence imaging. Invest Radiol 2011;46:187-195.

10. Deshpande N, Ren Y, Foygel K, Rosenberg J, Willmann JK. Tumor angiogenic marker expression levels during tumor growth: longitudinal assessment with molecularly targeted microbubbles and US imaging. Radiology 2011;258:804-811.

11. Willmann JK, Paulmurugan R, Chen K, Gheysens O, RodriguezPorcel M, Lutz AM, et al. US imaging of tumor angiogenesis with microbubbles targeted to vascular endothelial growth factor receptor type 2 in mice. Radiology 2008;246:508-518.

12. Ellegala DB, Leong-Poi H, Carpenter JE, Klibanov AL, Kaul S, Shaffrey ME, et al. Imaging tumor angiogenesis with contrast ultrasound and microbubbles targeted to alpha(v)beta3. Circulation 2003;108:336-341. 
13. Deckers R, Yudina A, Cardoit LC, Moonen CT. A fluorescent chromophore TOTO-3 as a 'smart probe' for the assessment of ultrasound-mediated local drug delivery in vivo. Contrast Media Mol Imaging 2011;6:267-274.
14. McDannold N, Arvanitis CD, Vykhodtseva N, Livingstone MS. Temporary disruption of the blood-brain barrier by use of ultrasound and microbubbles: safety and efficacy evaluation in rhesus macaques. Cancer Res 2012;72:3652-3663. 